\title{
COVID-19-induced anosmia associated with olfactory bulb atrophy
}

\author{
Andrew Chiu ${ }^{1}$ (D) Nancy Fischbein ${ }^{1} \cdot$ Max Wintermark ${ }^{1} \cdot$ Greg Zaharchuk $^{1} \cdot$ Paul T. Yun $^{2} \cdot$ Michael Zeineh $^{1}$
}

Received: 9 July 2020 / Accepted: 9 September 2020 / Published online: 15 September 2020

(C) Springer-Verlag GmbH Germany, part of Springer Nature 2020

\begin{abstract}
As the global COVID-19 pandemic evolves, our knowledge of the respiratory and non-respiratory symptoms continues to grow. One such symptom, anosmia, may be a neurologic marker of coronavirus infection and the initial presentation of infected patients. Because this symptom is not routinely investigated by imaging, there is conflicting literature on neuroimaging abnormalities related to COVID-19-related anosmia. We present a novel case of COVID-19 anosmia with definitive olfactory bulb atrophy compared with pre-COVID imaging. The patient had prior MR imaging related to a history of prolactinoma that provided baseline volumes of her olfactory bulbs. After a positive diagnosis of COVID-19 and approximately 2 months duration of anosmia, an MRI was performed that showed clear interval olfactory bulb atrophy. This diagnostic finding is of prognostic importance and indicates that the olfactory entry point to the brain should be further investigated to improve our understanding of COVID infectious pathophysiology.
\end{abstract}

Keywords COVID-19 $\cdot$ Anosmia $\cdot$ Olfactory bulb

\section{Main text}

Although hyposmia or anosmia has been reported in as many as $60 \%$ of patients who are symptomatic with COVID-19, imaging of the olfactory nerve is not routinely employed. The olfactory nerve is small and only well seen on dedicated

Andrew Chiu

ac3@stanford.edu

Nancy Fischbein

nancyfb@stanford.edu

Max Wintermark

mwinterm@stanford.edu

Greg Zaharchuk

gregz@stanford.edu

Paul T. Yun

pyun@stanfordhealthcare.org

Michael Zeineh

mzeineh@stanford.edu

1 Division of Neuroradiology, Department of Radiology, Stanford University, 300 Pasteur Drive, Room S047,

Stanford, CA 94305-5105, USA

2 Menlo Medical Clinic Stanford HealthCare, 321 Middlefield Road, 1st Floor, Menlo Park, CA 94205, USA skull base magnetic resonance imaging (MRI), so prospective assessments of its changes have been lacking. We present a case of a COVID-19 patient with anosmia showing atrophy of the olfactory bulbs by MRI in comparison with a presymptomatic MRI obtained for an unrelated cause.

A 19-year-old female presented to the otolaryngologist with persistent anosmia after a RT-PCR and serological confirmed diagnosis of COVID-19 1 month prior. At the time of presentation, the patient had a negative RT-PCR and resolution of COVID-19-related respiratory symptoms, but had not recovered her sense of olfaction. The patient underwent a skull base MRI, approximately 2 months after the onset of anosmia. Because of a history of prolactinoma treated medically, the patient had multiple prior MRIs over the course of 4 years. Compared with these multiple prior MRIs, visual and quantitative assessment of a coronal T2 fat-suppressed sequence through the anterior cranial fossa showed new bilateral atrophy of the olfactory bulbs (Fig. 1). The patient's most recent olfactory bulb volumes measured 3 years before COVID-19induced anosmia were $49 \cdot 5 \mathrm{~mm}^{3}$ and $47.46 \mathrm{~mm}^{3}$. In comparison, the olfactory bulb volumes on the MRI after COVID-19induced anosmia were $29.96 \mathrm{~mm}^{3}$ and $35.51 \mathrm{~mm}^{3}$, smaller than the minimum olfactory bulb volume in the literature of $54 \mathrm{~mm}^{3}$ in women $<45$ years of age [1].

Olfactory bulb volume loss in patients within the broad category of post-infectious anosmia is correlated with the 

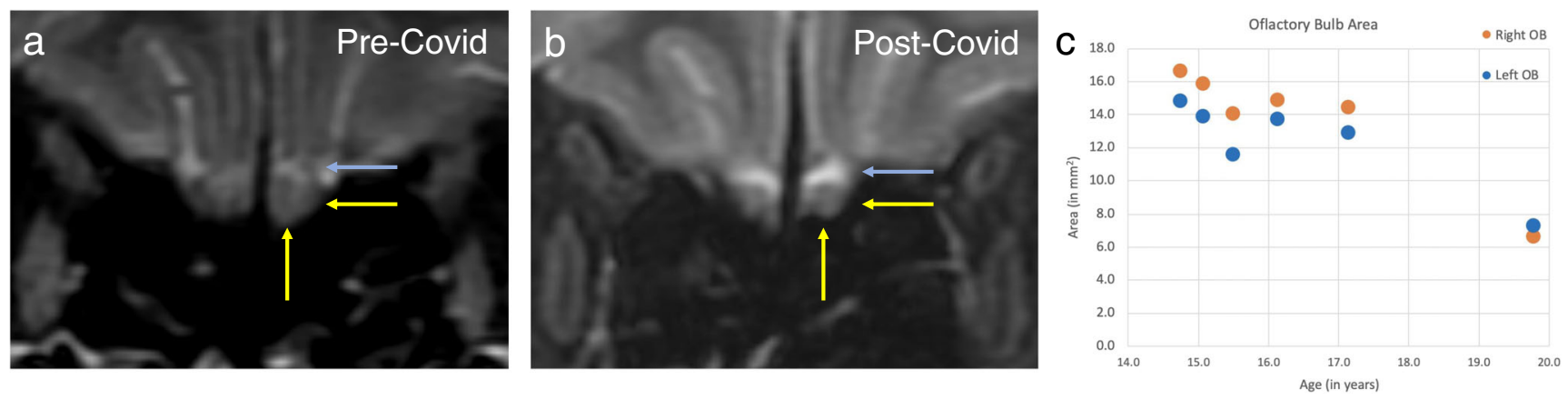

Fig. 1 Coronal T2 fat-suppressed 3-mm thick images $\mathbf{a}$ before and $\mathbf{b}$ after diagnosis of COVID-19. Notice the smaller size of olfactory bulbs (anatomic left in yellow arrows) within the olfactory grooves, as evidenced by

duration of olfactory loss $[2,3]$. Although the exact pathogenesis of COVID-19-induced anosmia has not been definitively elucidated, Brann et al. suggest that anosmia and dysgeusia in COVID-19 patients may be due to viral infection of the olfactory epithelial support cells such as the sustentacular cells and Bowman's gland cells [4]. Our results build upon recent imaging findings of the acute inflammatory phase of COVID-19induced anosmia by showing definitive atrophy compared with baseline imaging [5].

This case highlights a neuroimaging finding in patients with SARS-CoV-2 infection described only once before in the literature [5], with the novel addition of pre-COVID imaging confirming definitive atrophy. A limitation of this case is that the patient did not undergo formal psychophysical testing of her olfactory function. Olfactory bulb atrophy visualized on MR imaging occurs significantly more in patients with objectively measured olfactory dysfunction of various etiologies [6], but currently there are no studies assessing the correlation of olfactory bulb atrophy and objective olfactory tests specifically in COVID-19 patients. Future studies may evaluate changes in olfactory bulb volume in larger cohorts of COVID-19 patients at multiple timepoints with supplementary objective psychophysical olfactory function testing. MR imaging may then allow clinicians to provide patients with a more accurate prognosis regarding olfaction.

Funding No funding was received for this study.

\section{Compliance with ethical standards}

Conflict of interest The authors declare that they have no conflict of interest. Specifically, Dr. Chiu, Dr. Fischbein, Dr. Zaharchuk, and Dr. Zeineh have nothing to disclose. Dr. Yun reports personal fees from Intersect ENT, outside the submitted work. increased CSF (blue arrows) above the nerve. $\mathbf{c}$ Timecourse of patient's olfactory bulb size over 5 years, with a pronounced decrease on the most recent timepoint on the far right, occurring after diagnosis of COVID-19

Ethical approval All procedures performed in the studies involving human participants were in accordance with the ethical standards of the institutional and/or national research committee and with the 1964 Helsinki Declaration and its later amendments or comparable ethical standards. More specifically, an institutional Retrospective IRB was used.

Informed consent Informed consent was obtained from the patient for publication of images in this short report.

\section{References}

1. Buschhüter D, Smitka M, Puschmann S, Gerber JC, Witt M, Abolmaali ND, Hummel T (2008) Correlation between olfactory bulb volume and olfactory function. Neuroimage 42(2):498-502

2. Rombaux P, Mouraux A, Bertrand B, Nicolas G, Duprez T, Hummel $\mathrm{T}$ (2006) Olfactory function and olfactory bulb volume in patients with postinfectious olfactory loss. Laryngoscope 116:436-439

3. Yao L, Yi X, Pinto JM, Yuan X, Guo Y, Liu Y, Wei Y (2018) Olfactory cortex and olfactory bulb volume alterations in patients with post-infectious olfactory loss. Brain Imaging Behav 12:13551362

4. Brann DH, Tsukahara T, Weinreb C et al (2020) Non-neuronal expression of SARS-CoV-2 entry genes in the olfactory system suggests mechanisms underlying COVID-19-associated anosmia. BioRxiv published online May 18. https://doi.org/10.1101/2020.03. 25.009084(preprint)

5. Politi LS, Salsano E, Grimaldi M (2020) Magnetic resonance imaging alteration of the brain in a patient with coronavirus disease 2019 (COVID-19) and anosmia. JAMA Neurol published online May 29. https://doi.org/10.1001/jamaneurol.2020.2125

6. Chung MS, Choi WR, Jeong HY, Lee JH, Kim JH (2018) MR Imaging-based evaluations of olfactory bulb atrophy in patients with olfactory dysfunction. AJNR Am J Neuroradiol 39(3):532-537

Publisher's note Springer Nature remains neutral with regard to jurisdictional claims in published maps and institutional affiliations. 